\title{
NORMALIZATION OF THE PARAMETERIZED COURANT-SNYDER MATRIX FOR SYMPLECTIC FACTORIZATION OF A PARAMETERIZED TAYLOR MAP
}

\author{
Yiton T. Yan \\ Superconducting Super Collider Iaboratory*, \\ 2550 Beckleymeade Avenue, Dallas, TX 75237
}

\section{Abstract}

The transverse motion of charged particles in a circular accelerator can be well represented by a one-turn highorder Taylor map [1]. For particles without energy deviation, the one-turn Taylor map is a 4-dimensional polynomials of four variables. The four variables are the transverse canonical coordinates and their conjugate momenta. To include the energy deviation (off-momentum) effects, the map has to be parameterized with a smallness factor representing the off-momentum and so the Taylor map becomes a 4-dimensional polynomials of five variables. It is for this type of parameterized Taylor map that a mehtod is presented for converting it into a parameterized Dragt-Finn facctorization map. Parameterized nonlinear normal form and parameterized kick factorization can thus be obtained with suitable modification of the existing technique.

\section{INTRODUCTION}

A one-turn Taylor map parameterized for off-momentum transverse propagation relative to the dispersed closed orbit in a circular alternating-gradient synchrotron [2] can be given in the form

$$
\begin{aligned}
\overrightarrow{x^{\prime}} & =m(\vec{x}, \delta): \vec{x} \\
& =M(\delta) \vec{x}+\sum_{i=0}^{n_{2}} \vec{U}_{2, i}(\vec{x}) \delta^{i}+\sum_{i=0}^{n_{3}} \vec{U}_{3, i}(\vec{x}) \delta^{i}+\cdots,(1)
\end{aligned}
$$

where the transverse canonical phase space coordinates, $\vec{x}=\left[x, p_{x}, y, p_{y}\right]$ are deviations relative to the dispersed closed orbit; $\delta=\Delta P / P_{0}$ is a parameter for the offmomentum, which is usually a smallness factor in the domain of physical interest. Terms in each of the polynomials $\vec{U}_{k, i}(\vec{x})$ for $k=2,3, \cdots$ and $i=0,1, \cdots, n_{k}$ are all of the order $k$ of $\vec{x}$. The parameterized Courant-Snyder matrix, $M(\delta)$, is truncated at an order of $\delta^{n}$ and given by

$$
M(\delta)=M_{0}+\delta M_{1}+\delta^{2} M_{2}+\cdots+\delta^{n} M_{n},
$$

where $M_{0}, M_{1}, \cdots, M_{n}$ are all $4 \times 4$ and usually coupled matrices. Note that $n \geq n_{2} \geq n_{3} \geq \cdots \geq n_{k} \geq \cdots$ and that the dispersed closed orbit can be expressed as

$$
\vec{x}_{c}=\vec{x}_{c, 0}+\delta \vec{x}_{c, 1}+\delta^{2} \vec{x}_{c, 2}+\cdots,
$$

** Operated by Universities Research Association, Inc., for the U.S. Department of Energy under the Contract No. DE-AC0289ER40486.

U.S. Government work not protected by U.S. Copyright. which is a polynomial of $\delta$ with an order of $\mathrm{n}$ or higher.

If the Taylor map is extracted from a symplectic tracking program, then it is symplectic when it is expanded to the infinite order of $\vec{x}$ and $\delta$. We assume the Taylor map given by Eq. 1 is such a type of Taylor map truncated at a finite order of $\delta$ and $\vec{x}$. Such a truncated Taylor map, although not symplectic because of the missing of the high-order effects, can be order-by-order converted into a Dragt-Finn factorization map [3]. However, the addition of the parameter $\delta$ makes such a factorization process trickier. In this paper, the author presents a method of factorizing such a perameterized Taylor map given by Eq. 1. Only a single parameter, $\delta$, is considered though extension to a multiparameter case is analogous.

\section{Normalization Of $M(\delta)$}

The key to the Dragt-Finn factorization of a parameterized Taylor map given by Eq. 1 is the normalization of its associated parameterized Courant-Snyder matrix $M(\delta)$ given by Eq. 2. A method [4] has been presented recently to normalize $M(\delta)$ via order-by-order symplectic factorization such that

$$
R(\delta)=A^{-1}(\delta) M(\delta) A(\delta)+\sigma\left(\delta^{n+1}\right),
$$

where the normalized parameterized rotation $R(\delta)$ and the canonical generation matrix $A(\delta)$ are given as follows.

$$
R(\delta)=\left(\begin{array}{cccc}
\cos \mu_{1}(\delta) & \sin \mu_{1}(\delta) & 0 & 0 \\
-\sin \mu_{1}(\delta) & \cos \mu_{1}(\delta) & 0 & 0 \\
0 & 0 & \cos \mu_{2}(\delta)^{\cdot} & \sin \mu_{2}(\delta) \\
0 & 0 & -\sin \mu_{2}(\delta) & \cos \mu_{2}(\delta)
\end{array}\right)
$$

where

$$
\mu_{\alpha}(\delta)=\mu_{\alpha, n}+\delta \mu_{r, 1}+\delta^{2} \mu_{\alpha, 2}+\cdots+\delta^{n} \mu_{\alpha, n},
$$

for $\alpha=1,2$. The inverse of $R(\delta)$ is also a decoupled rotation given by

$$
R^{-1}(\delta)=\left(\begin{array}{cccc}
\cos \mu_{1}(\delta) & -\sin \mu_{1}(\delta) & 0 & 0 \\
\sin \mu_{1}(\delta) & \cos \mu_{1}(\delta) & 0 & 0 \\
0 & 0 & \cos \mu_{2}(\delta) & -\sin \mu_{2}(\delta) \\
0 & 0 & \sin \mu_{2}(\delta) & \cos \mu_{2}(\delta)
\end{array}\right)
$$

The symplectic matrix $A(\delta)$ is of order $\delta^{n(n+1) / 2}$ which is a concatenation of a series order-by-order canonical generation matrices given by

$$
A(\delta)=A_{0}\left(I+\delta^{1} A_{1}\right)\left(I+\delta^{2} A_{2}\right) \cdots\left(I+\delta^{n} A_{n}\right) .
$$


Its inverse is given by

$$
A^{-1}(\delta)=\left(I-\delta^{n} A_{n}\right) \cdots\left(I-\delta^{2} A_{2}\right)\left(I-\delta A_{1}\right) A_{0}^{-1} .
$$

Note that $A_{0}$ and its inverse $A_{0}^{-1}$ are the familiar generation matrices for normalization of $M_{0}$ such that [5]

$$
\begin{aligned}
R_{0} & =A_{0}^{-1} M_{0} A_{0} \\
& =\left(\begin{array}{cccc}
\cos \mu_{1,0} & \sin \mu_{1,0} & 0 & 0 \\
-\sin \mu_{1,0} & \cos \mu_{1,0} & 0 & 0 \\
0 & 0 & \cos \mu_{2,0} & \sin \mu_{2,0} \\
0 & 0 & -\sin \mu_{2,0} & \cos \mu_{2,0}
\end{array}\right) .
\end{aligned}
$$

For the detailed solution of $\mu_{\alpha, i}$ and $A_{i}$ for $i=1,2, \cdots, n$ and $\alpha=1,2$, please refer to Reference [4].

\section{Dragt-Finn Factorization}

For Dragt-Finn factorization of the parameterized Taylor map given by Eq. 1, one first makes a similarity transformation on the map such that

$$
\begin{aligned}
{ }_{1} m(\vec{x}, \delta): \vec{x} & =\mathcal{A}(\vec{x}, \delta) m(\vec{x}, \delta) \mathcal{A}^{-1}(\vec{x}, \delta): \vec{x} \\
& =R(\delta) \vec{x}+\sum_{i=0}^{n_{2}} \vec{U}_{2, i}(\vec{x}) \delta^{i}+\sum_{i=0}^{n_{3}} \vec{U}_{3, i}(\vec{x}) \delta^{i}+\cdots,
\end{aligned}
$$

where $\mathcal{A}(\vec{x}, \delta)$ is the global form of $A(\delta)$. One then make a concatenation of the transformed map ${ }_{1} m(\vec{x}, \delta)$ with $\mathcal{R}^{-1}(\vec{x}, \delta)$ such that

$$
\begin{aligned}
{ }_{2} m(\vec{x}, \delta): \vec{x} & =\mathcal{R}^{-1}(\vec{x}, \delta){ }_{1} m(\vec{x}, \delta): \vec{x} \\
& =\vec{x}+\sum_{i=0}^{n_{2}}{ }_{2} \vec{U}_{2, i}(\vec{x}) \delta^{i}+\sum_{i=0}^{n_{3}} \vec{U}_{3, i}(\vec{x}) \delta^{i}+\cdots,(4)
\end{aligned}
$$

where $\mathcal{R}^{-1}(\vec{x}, \delta)$ is the global form of the inverse of the rotation matrix $R(\delta)$. Due to symplecticity of the Taylor map (when it is expanded to the infite order), for each of the ${ }_{2} \vec{U}_{2, i}(\vec{x})$ for $i=0,1, \cdots, n_{2}$ there exists a third-order (every term is third order) polynomial of $\vec{x}, f_{3, i}(\vec{x})$ such that

$$
\left[f_{3, i}(\vec{x}), \vec{x}\right]={ }_{2} \vec{U}_{2, i}(\vec{x})
$$

Therefore, from Eq. 4, one obtains

$$
{ }_{2} m(\vec{x}, \delta): \vec{x}=\exp \left(: \sum_{i=0}^{n_{2}} f_{3, i}(\vec{x}) \delta^{i}:\right) \vec{x}+\sum_{i=0}^{n_{3}} \vec{U}_{3, i}(\vec{x}) \delta^{i}+\cdots
$$

where

$$
{ }_{2} \vec{U}^{\prime}{ }_{3, i}(\vec{x})={ }_{2} \vec{U}_{3, i}(\vec{x})-\frac{1}{2}\left[f_{3, i}(\vec{x}),\left[f_{3, i}(\vec{x}), \vec{x}\right]\right]
$$

Concatenating $2 m(\vec{x}, \delta)$ with

$$
\exp \left(-: \sum_{i=0}^{n_{2}} f_{3, i}(\vec{x}) \delta^{i}:\right)
$$

one obtains

$$
\begin{aligned}
{ }_{3} m(\vec{x}, \delta): \vec{x} & =\exp \left(-: \sum_{i=0}^{n_{2}} f_{3, i}(\vec{x}) \delta^{i}:\right)_{2} m(\vec{x}, \delta): \vec{x} \\
& =\vec{x}+\sum_{i=0}^{n_{3}}{ }_{3} \vec{U}_{3, i}(\vec{x}) \delta^{i}+\sum_{i=0}^{n_{4}}{ }_{3} \vec{U}_{4, i}(\vec{x}) \delta^{i}+\cdots
\end{aligned}
$$

Again, due to symplecticity, for each of the ${ }_{3} \vec{U}_{3, i}(\vec{x})$ for $i=0,1, \cdots, n_{3}$, there exists a fourth-order (every term is fourth order) polynomial of $\vec{x}, f_{4, i}(\vec{x})$ such that

$$
\left[f_{4, i}(\vec{x}), \vec{x}\right]={ }_{3} \vec{U}_{3, i}(\vec{x}) .
$$

One thus obtains the Dragt-Finn factorization of the fourth order given by

$$
\exp \left(: \sum_{i=0}^{n_{3}} f_{4, i}(\vec{x}) \delta^{i}:\right)
$$

Following the similar steps given above, one can proceed further to obtain the Dragt-Finn factorization of the higher orders given by

$$
\exp \left(: \sum_{i=0}^{n_{k-1}} f_{k, i}(\vec{x}) \delta^{i}:\right)
$$

for $k=5,6, \cdots$. The parameterized Taylor map given by Eq. 1 can then be represented by a symplectic map given in global form by

$$
\begin{aligned}
\overrightarrow{x^{\prime}} & =m(\vec{x}, \delta): \vec{x} \\
& =\mathcal{A}^{-1}(\vec{x}, \delta) m_{f}(\vec{x}, \delta) \mathcal{A}(\vec{x}, \delta): \vec{x}
\end{aligned}
$$

where

$m_{f}(\vec{x}, \delta)=\mathcal{R}(\vec{x}, \delta) \exp \left(: \sum_{i=0}^{n_{2}} f_{3, i}(\vec{x}) \delta^{i}:\right) \exp \left(: \sum_{i=0}^{n_{3}} f_{4, i}(\vec{x}) \delta^{i}:\right) \cdots$

\section{Discussions}

At first glance, one may wonder why Eq. 5 (or Eq. 6), which is one of the important steps for leading to the parameterized Dragt-Finn factorization of a dispersed-closedorbit Taylor map, is true. Let us assume that the Taylor expansions are all infinite orders of $\delta$, that is, $n=n_{2}=$ $n_{3}=\cdots=\infty$. If a suitable small factor is substituted for $\delta$ into Eq. 1, then one obtains the associated regular closed-orbit non-parametrized Taylor map which is a familiar type of map for Dragt-Finn factorization. Now if we first obtain the linear parameterized normalized rotation $R(\delta)$ and its associated parametrized canonical generation matrix $A(\delta)$ up to the infinite order of $\delta$, and then substitute the same small factor for $\delta$, we would have obtained the same linear non-parameterized normalized rotation $R$ and its associated non-parametrized canonical generation matrix $A$ as those obtained for the associated 
non-parameterized Dragt-Finn factorization. Therefore, it would make no differnece whether we first obtain Eq. 4 to infinite order of $\delta$ and then substitute the small factor for $\delta$ or we first substitute the small factor for $\delta$ in Eq. 1 to obtain a non-parameterized closed-orbit Taylor map and then obtain the associated non-parameterized equivalence of Eq. 4. Thus, due to symplecticity, there must exist a third-order (every term is third order) polynomial of $\vec{x}$, $f(\vec{x}, \delta)$, such that

$$
[f(\vec{x}, \delta), \vec{x}]=\sum_{i=0}^{\infty}{ }_{2} \vec{U}_{2, i}(\vec{x}) \delta^{i} .
$$

Since $f(\vec{x}, \delta)$ can always be Taylor expanded as

$$
f(\vec{x}, \delta)=\sum_{i=0}^{\infty} f_{i}(\vec{x}) \delta^{i},
$$

by comparing Eq. 8 and Eq. 9, one can conclude that

$$
\left[f_{i}(\vec{x}), \vec{x}\right]={ }_{2} \vec{U}_{2, i}(\vec{x})
$$

for cach $i=0,1,2, \cdots, \infty$. Furthermore, since terms with a certain order of $\delta$ can only be contributed through concatenation from terms with lower or equal orders of $\delta$, truncation of higher-order terms will not change the outcome for the lower-order Lie operators. Therefore, $f_{3, i}(\vec{x})=f_{i}(\vec{x})$ for $i=0,1, \cdots, n_{2}$.

In practice, differential algebra [6] can be used to obtain the Taylor map. Indeed, a truncated Taylor map of the type given by Eq. 1 and its associated dispersed closed orbit given by Eq. 3 can be obtained for the SSC lattice (and others) with the use of a post-Teapot [7] map extraction program Zmap [8]. Implementation of such a parameterized Taylor-map Dragt-Finn factorization can be achieved with some modification of the available nonparameterized Dragt-Finn factorization routine in Zlib [9] and others [10] [11]. Once the parameterized dragt-Finn factorization of the type given by Eq. 7 is obtained, paramterized normal form can be obtained with suitable modification of the existing technique [10] and parameterized kick factorization [12] [13] can be obtained for faster longterm symplectic kick map tracking.

\section{ACKNOWLEDGMENTS}

The author thanks A. Chao for his support and encouragement and J. Ellison, E. Forest, and J. Irwin for many discussions.

\section{REFERENCES}

[1] Y. Yan, T. Sen, A. Chao, G. Bourianoff, A. Dragt, and E. Forest, SSC Laboratory Report SSCL-301 (1990); also to appear in the proceedings of the workshop on nonlinear problems in future particle accelerators, Capri, Italy (1990).

[2] E. Courant and H. Snyder, Ann. Phys., 3, 1 (1958)
[3] A. J. Dragt and J. M. Finn, J. Math. Phys. 20, 2649 (1979)

[4] Y. Yan, SSC Laboratory Report SSCL-302 (1990).

[5] D. Edwards and L. Teng, in Proceedings of the 1973 IEEE Particle Accelerator Conference, p. 885 (1973); L.C. Teng, Fermi National Laboratory Report FN-229, 1971.

[6] M.Berz, in Floyd Bennett and Joyce Kopta, editors, Proceedings of the 1989 IEEE Particle Accelerator Conference, March 20-23, 1989, IEEE Catalog Number $89 \mathrm{CH} 2669-0$.

[7] L. Schachinger and R. Talman, Particle Accelerators, Vol. 22, 1987.

[8] Y. Yan, SSC Laboratory Report SSCL-299, 1990.

[9] Y. Yan and C. Yan, SSC Laboratory Report SSCL-300 (1990)

[10] E. Forest, M. Berz, and J. Irwin, Particle Accel. 24, 91 (1989), and M. Berz, ibid. 24, 109 (1989)

[11] L. Michelotti, in these Proceedings, and Fermi National Accelerator Laboratory Report FN-535, January 31 (1990)

[12] J. Irwin, SSC Laboratory Report SSCL-228 (1990).

[13] A. J. Dragt, to appear in the proceedings of the workshop on nonlinear problems in future particle accelerators, Capri, Italy (1990). 